\title{
Quasiparticle Interference in a Superconducting State with a Fourfold Phase Change
}

\author{
P. Pisarski and G. Harań* \\ Institute of Physics, Wrocław University of Technology \\ Wybrzeże Wyspiańskiego 27, 50-370 Wrocław, Poland
}

Dedicated to Professor Józef Spatek on the occasion of his 60th birthday

Quantum interference revealed in $\mathrm{Bi}_{2} \mathrm{Sr}_{2} \mathrm{CaCu}_{2} \mathrm{O}_{8+\delta}$ by Fourier transform scanning spectroscopy is primarily determined by maxima of the joint density of states which correspond to an octet of scattering wave vectors connecting points at the constant quasiparticle energy contours where the density of states is maximal. We consider a superconductor with a constant quasiparticle density of states where the interference is due to the fourfold change in the phase of the order parameter. Our results may be helpful in a discussion of the interference in a $d$-wave superconductor at the tunneling energy exceeding the gap magnitude.

PACS numbers: 74.20.Rp, 74.25.Jb, 74.62.Dh

\section{Introduction}

Scanning tunneling microscopy (STM) [1,2] and Fourier transform scanning tunneling microscopy (FT STM) [3, 4] provide a significant piece of information on the quasiparticle spectrum of the superconducting state in cuprates. The FT STM results for $\mathrm{Bi}_{2} \mathrm{Sr}_{2} \mathrm{CaCu}_{2} \mathrm{O}_{8+\delta}$ have been interpreted within the $d$-wave superconductivity scenario as the quasiparticle scattering interference induced by disorder with the interference pattern determined by the octet vectors connecting points at the constant quasiparticle energy contours where the density of states is maximal $[4,5]$. In our report we point out to the significance of a change of a superconducting phase in establishing the interference images.

\section{Theory}

In order to focus on the quasiparticle interference driven solely by a varying superconducting phase and to exclude the effect of $k$-spots with a high density of

*corresponding author; e-mail: haran@pwr.wroc.pl 
states we employ a model superconducting state with a $d$-wave-like phase change and a uniform quasiparticle density of states along a constant energy contour. We consider a superconducting gas with a two-dimensional order parameter of a fourfold phase change $[6]$

$$
\Delta_{k}=\Delta \operatorname{sgn}(\cos 2 \phi)=\Delta \operatorname{sgn}\left(k_{x}^{2}-k_{y}^{2}\right)
$$

where $\phi$ is a polar angle of a momentum vector $\boldsymbol{k}=\left(k_{x}, k_{y}\right)$. At a fixed and constant energy $\omega$ the quasiparticle excitations of a superconducting state form contours of constant quasiparticle energy (CCE) determined by the dispersion $E_{k}= \pm \sqrt{\varepsilon_{k}^{2}+\Delta_{k}^{2}}$. For the assumed parabolic spectrum in a normal state, $\varepsilon_{k}=k^{2} / 2 m-\mu$, where $\mu=k_{\mathrm{F}}^{2} / 2 m$ is the Fermi energy, and $m$ is the quasiparticle mass, the CCEs consist of two concentric circles [7] of radii $k_{ \pm}=k_{\mathrm{F}}\left(1 \pm \sqrt{\omega^{2}-\Delta^{2}} / \mu\right)^{1 / 2}$. The quasiparticle density of states is locally proportional to $\left|\nabla E_{k}\right|^{-1}$, which for the superconducting state (1) reads

$$
\left|\nabla E_{\boldsymbol{k}_{ \pm}}\right|^{-1}=\frac{m \omega / \sqrt{\omega^{2}-\Delta^{2}}}{k_{\mathrm{F}}\left(1 \pm \sqrt{\omega^{2}-\Delta^{2}} / \mu\right)^{1 / 2}}
$$

and is constant along the CCEs. For such a superconducting state with a varying phase and a constant density of states we look at the quasiparticle interference around an isotropic scattering center of a potential $v$. We study the effect of the impurity potential on the Fourier-transformed local density of states $N(\boldsymbol{q}, \omega)$ in the linear approximation at zero temperature. $\delta N(\boldsymbol{q}, \omega)$ is defined as a Fourier transform of a local density of states $\delta N(\boldsymbol{r}, \omega)$

$$
\delta N(\boldsymbol{r}, \omega)=\sum_{\boldsymbol{q}} \mathrm{e}^{\mathrm{i} \boldsymbol{q} \boldsymbol{r}} \delta N(\boldsymbol{q}, \omega) .
$$

The impurity-induced change of $N(\boldsymbol{q}, \omega)$ for a point-like impurity potential $v$ is determined by the joint density of states [5]

$$
\delta N(\boldsymbol{q}, \omega)=-\frac{1}{\pi} \operatorname{Im}\left(\int \frac{\mathrm{d}^{2} k}{(2 \pi)^{2}} \hat{g}_{0}(\boldsymbol{k}+\boldsymbol{q}) v \hat{\tau}_{3} \hat{g}_{0}(\boldsymbol{k})\right)_{11},
$$

where $\hat{g}_{0}(\boldsymbol{k}, \omega)=\left(\mathrm{i} \omega \hat{\tau}_{0}-\varepsilon_{k} \hat{\tau}_{3}-\Delta_{\boldsymbol{k}} \hat{\tau}_{1}\right)^{-1}$. For the superconducting state $(1)$ the joint density of states separates into a symmetric part of an $s$-wave state $\delta N_{s}[7]$ and a phase-dependent function $\delta N_{\phi}$

$$
\delta N(\boldsymbol{q}, \omega)=\delta N_{s}(\boldsymbol{q}, \omega)+\delta N_{\phi}(\boldsymbol{q}, \omega)
$$

\section{Results}

Quasiparticle interference is mostly determined by the maxima of the joint density of states. For the $s$-wave superconductor they correspond to CCEs nesting wave vectors $2 k_{ \pm}$, which represent the difference in quasiparticles momenta within a Cooper pair. In the case of the varying phase of the order parameter we have additional interference peaks set by $\boldsymbol{q}_{i}$ vectors, which correspond to the scattering processes between the Cooper pairs, which differ in phase (Fig. 1). There are two kinds of scattering processes: (1) within a single CCE - vectors $\boldsymbol{q}_{1}$ 

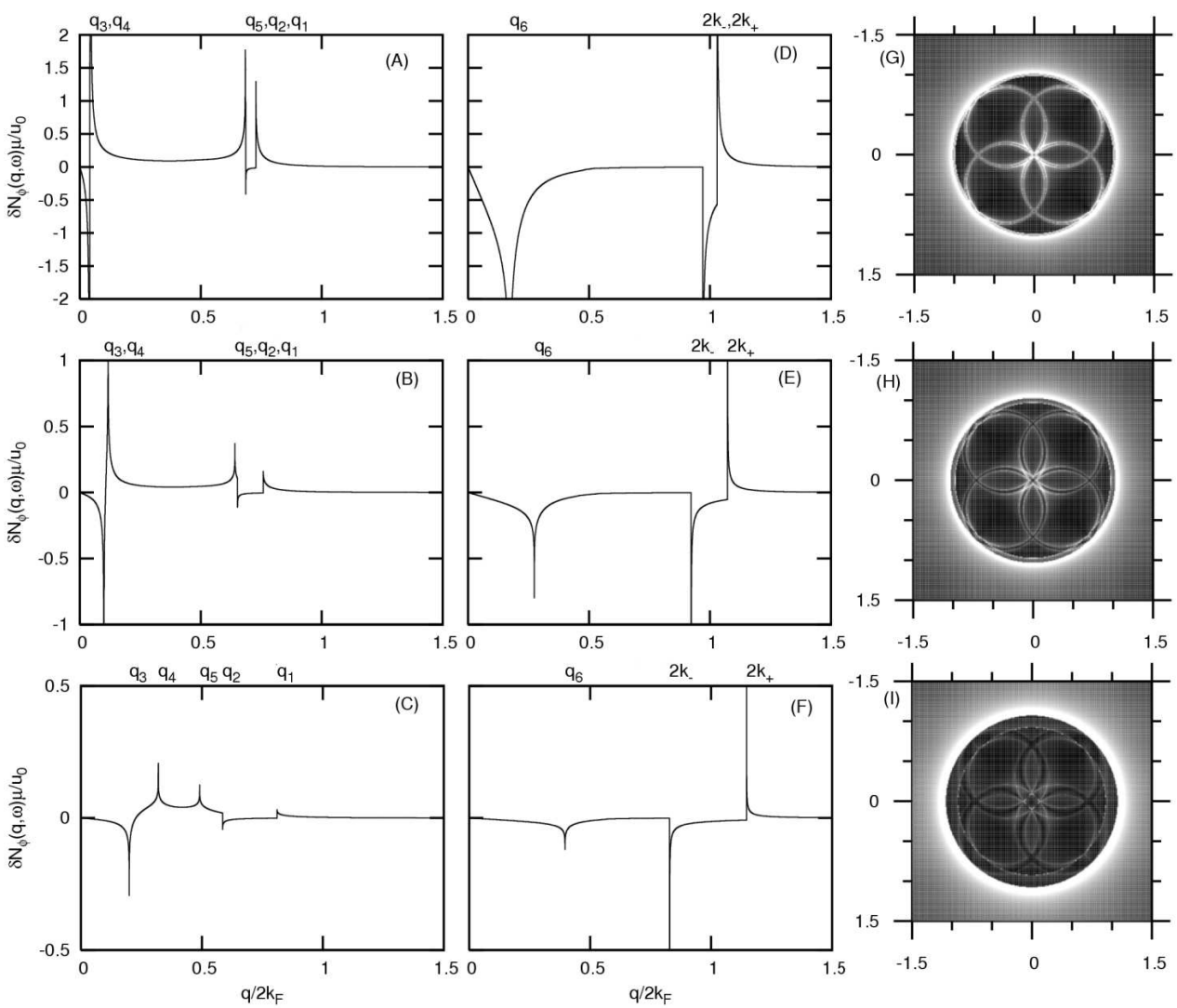

Fig. 1. Phase-dependent part $\delta N_{\phi}(\mathbf{q}, \omega)$ of a Fourier transformed local density of states (FTLDOS) for the q-vectors: [1,0] (first column) and [1,1] (second column). Third column: FTLDOS map in the $\left(q_{x}, q_{y}\right)$ plane for $\Delta / \mu=1 / 6 \pi$, white color represents large and black color - low values of $\delta N(\mathbf{q}, \omega)$. Momentum vectors are measured in $2 k_{\mathrm{F}}$ units, and the impurity potential is given by a dimensionless value $u_{0}=\pi N_{0} v$, where $N_{0}$ is a density of states for a single spin direction at the Fermi level. Tunneling energies: $\omega=$ $1.5 \Delta(\mathrm{A}, \mathrm{D}, \mathrm{G}), \omega=3 \Delta(\mathrm{B}, \mathrm{E}, \mathrm{H}), \omega=6 \Delta(\mathrm{C}, \mathrm{F}, \mathrm{I})$. Maxima of $\delta N_{\phi}(\mathbf{q}, \omega)$ correspond to scattering wave vectors: $q_{1}=\sqrt{2} k_{+}, q_{2}=\sqrt{2} k_{-}, q_{3}=\left(\sqrt{2 k_{+}^{2}-k_{-}^{2}}-k_{-}\right) / \sqrt{2}$, $q_{4}=\left(k_{+}-\sqrt{2 k_{-}^{2}-k_{+}^{2}}\right) / \sqrt{2}, q_{5}=\left(k_{+}+\sqrt{2 k_{-}^{2}-k_{+}^{2}}\right) / \sqrt{2}, q_{6}=\sqrt{k_{+}^{2}-k_{-}^{2}}$.

and $\boldsymbol{q}_{2},(2)$ between $k_{+}$and $k_{-}$CCEs $-\boldsymbol{q}_{i>2}$. In the third column of Fig. 1 we show the quasiparticle interference map around a point-like impurity for the superconducting state (1).

\section{Conclusion}

Concluding, we note a significant role of the phase change of the order parameter in the quasiparticle interference. Although the effect decreases with increasing excitation energy, it may be relevant in the interpretation of the Fourier- 
-transformed STM images of high-temperature superconductors at the tunneling energy slightly exceeding the gap magnitude.

\section{Acknowledgments}

The work is supported in part by the State Committee for Scientific Research grant no. 1P03B04630.

\section{References}

[1] S.H. Pan, E.W. Hudson, K.M. Lang, H. Eisaki, S. Uchida, J.C. Davis, Nature 403, 746 (2000).

[2] E.W. Hudson, K.M. Lang, V. Madhavan, S.H. Pan, H. Eisaki, S. Uchida, J.C. Davis, Nature 411, 920 (2001).

[3] J.E. Hoffman, K. McElroy, D.-H. Lee, K.M. Lang, H. Eisaki, S. Uchida, J.C. Davis, Science 295, 466 (2002).

[4] K. McElroy, R.W. Simmonds, J.E. Hoffman, D.-H. Lee, J. Orenstein, H. Eisaki, S. Uchida, J.C. Davis, Nature 442, 592 (2003).

[5] Q.-H. Wang, D.-H. Lee, Phys. Rev. B 67, 020511 (2003).

[6] G. Preosti, H. Kim, P. Muzikar, Phys. Rev. B 50, 1259 (1994).

[7] L. Capriotti, D.J. Scalapino, R.D. Sedgewick, Phys. Rev. B 68, 014508 (2003). 